\title{
When Is It Safe to See the Doctor?
}

\author{
Dianne L. Goede, MD (1), Melanie G. Hagen, MD, Senthil Meenrajan, MD, and \\ Margaret C. LO, MD
}

Department of Medicine, Division of Internal Medicine, University of Florida College of Medicine, Gainesville, FL, USA.

J Gen Intern Med 35(10):3071-2

DOI: $10.1007 / \mathrm{s} 11606-020-06034-3$

(c) Society of General Internal Medicine 2020

$\mathrm{W}$ hen the COVID19 pandemic struck the United States and closed all non-essential businesses, the medical world was thrust into turmoil. Suddenly, visiting your doctor was considered unsafe. Emergency room visits and hospital admissions plummeted in regions that were not COVID19 hot spots. Clinic volumes dropped and physicians grappled to navigate an upended healthcare system. Medicare shifted its policy toward telemedicine care for both COVID19 and nonCOVID19-related concerns. In response, healthcare systems drastically changed their protocols related to triaging, scheduling, documentation, and billing. In our primary care practice, telemedicine became the default visit type for both acute and chronic care issues to mitigate the COVID19 spread and shelter our high-risk patients. Yet, we must take heed of the psychological stress to patients. Every visit conversion to telemedicine has the potential to send the message: "it is not safe to visit your doctor." Such psychologic fear may drive patients to delay care for acute complaints and uncontrolled chronic diseases, further worsening morbidity and mortality during COVD19. ${ }^{1}$ The reality is that COVID19 is here to stay. We, clinicians, must now determine the appropriate use of telemedicine for acute and chronic care management during a public health crisis. We must now partner with our public health experts to refine guidelines and procedures to ensure the safety of face-to-face visits. Inevitably, our patients will ask us, "Should I come to clinic or stay safe at home?"

Telemedicine, telehealth, and related terms refer to the exchange of medical information from one site to another through electronic communication to improve a patient's health. Studies demonstrate telemedicine's effectiveness on chronic disease management, patient satisfaction, and economic impact. ${ }^{2,} 3$ These include hypertension monitoring, mental health evaluation and treatment, diabetes control, and dermatologic evaluation. ${ }^{3}$ The American College of Physicians supports the use of telemedicine in appropriate

Received June 11, 2020

Accepted July 1, 2020

Published online July 23, 2020 circumstances and cites its benefit in the setting of established patient-physician relationships. ${ }^{4}$ Our faculty early adopters indeed voice novel benefits of telemedicine care. Telemedicine brings the physician into the patients' home, revealing useful information about their living environment. Medication reconciliation is smoother and more accurate when patients can show their medications over video. We can consult and educate family members without multiple people in clinic. Undoubtedly, telemedicine allows us to learn more about our patients and help them in unique ways.

However, telemedicine was never intended to be the default way we provide care. Pre-COVID, telemedicine use was limited due to financial, time, and safety concerns. Most legislation on telemedicine ensures the same standard of care is provided when delivering care via telemedicine as care provided in face-to-face visits. Physicians are responsible for determining the safety and appropriateness of telemedicine visits for their individual patients. Yet, during this pandemic, we are tasked to expand telemedicine care outside of evidencebased practices with limited training. Although certain chronic diseases and cancer screenings can be addressed over telemedicine, acute complaints (e.g., angina, abdominal pain) and preventative health visits necessitate face-to-face interactions for a thorough clinical evaluation, physical examination, and administration of medications or immunizations. We must also tackle the potential healthcare disparity for patients without access to or skills in televideo technology. These individuals tend to be vulnerable populations already handicapped by social determinants of health needs and can be inadvertently harmed by telemedicine expansion.

The limitations of telemedicine have motivated us to implement interventions that enhance patient safety during faceto-face visits in the COVID19 era. We can learn from countries that rapidly contained the spread of SARS-CoV-1 infection through stringent infection control policies. ${ }^{5}$ The latest CDC infection control guidance for outpatient settings reflects many of these strategies learned. ${ }^{6}$ Early adoption of these strategies by ambulatory practices is essential for the safe return of face-to-face visits:

- Implement algorithms to identify patients with COVID19 symptoms and appropriate triage and use of telehealth to the level of care needed.

- Screen all patients, visitors, and healthcare workers entering facility for fever and COVID19 symptoms. 
- Require all patients, visitors, and healthcare workers to always wear a facemask while in the facility for source control.

- Designate a sick area to separate patients with respiratory or other symptoms from healthy individuals.

- Strategically communicate safety measures to patients through visual alerts (e.g., signs and posters at entrances).

- Ensure patients are at least 6 feet apart in waiting areas.

- Institute curbside check-in and/or waiting in which scheduled patients remain outside (e.g., stay in their vehicle) until the clinician is ready to see them in clinic.

- Implement sick leave policies that are non-punitive, flexible, and consistent with public health policies for ill healthcare workers to stay home.

Balancing the risks and benefits of face-to-face visits is a novel challenge. Early in the pandemic, the risk of COVID19 exposure during face-to-face visits was unknown due to the lack of testing ability and minimal knowledge about the methods of virus transmission. More data is now available from the CDC and local health departments to help physicians determine COVID19 transmission in their community and the potential for patient harm. ${ }^{7}$ With the risk of face-to-face visits differing throughout the United States, we must monitor these resources closely and work with our local health departments to determine the safety of face-toface visits specific to each physician's local practice. Physicians need to stay well-informed of their local COVID19 trends and communicate to patients all implemented infection control measures appropriate to their healthcare setting. Ways to deliver such communication are dependent on the individual clinic workflow but can include local media announcements, secure messaging, direct mailings, and/or staff-directed patient calls. Now more than ever, open communication and active shared decision-making between patients and physicians are essential to determine the appropriate type of visit to safely manage their medical needs. Prioritizing care to at-risk populations and those who may incur harm from care delays will help to lessen morbidity and mortality during this public health crisis.

Expanded telemedicine use is likely here to stay for many regions of the USA. To help physicians navigate this "new normal," we propose:

- Health systems develop protocols to ensure safe triaging and scheduling of telemedicine visits for appropriate complaints.

- Professional and accrediting bodies establish telemedicinespecific competency-based skills for physicians.

- Increase faculty development and CME training for telemedicine healthcare delivery.
- Develop innovative tools for virtual patient outreach and education, e.g., video-teleconference group education on exercise health, nutrition, etc.

- Robust clinical trials to determine the efficacy of telemedicine delivery compared with face-to-face visits for the management of common ambulatory health conditions.

As we progress toward the "backside of the curve," let us use the challenges of COVID19 as an opportunity to balance telemedicine care with the safe return of in-person visits for our patients. To do so, we must refine our skills in telemedicine delivery, implement stringent healthcare protocols for disease mitigation and safe triaging, communicate safety measures to our patients, and engage our patients in shared decision-making on the appropriate visit types to manage their medical needs. Such interventions will not only reduce the spread of COVID19 but also send a positive message to our patients that "yes, it is safe to see your doctor."

Corresponding Author: Dianne L. Goede, MD; Department of Medicine, Division of Internal Medicine, University of Florida College of Medicine, Gainesville, FL, USA (e-mail: dianne.roberts@medicine. ufl.edu).

\section{Compliance with Ethical Standards:}

Conflict of Interest: The authors declare that they do not have a conflict of interest.

\section{REFERENCES}

1. De Filippo O, D'Aso F, Angelini F, Bocchino PP, Conrotto F, Saglietto A, et al. Reduced Rate of Hospital Admissions for ACS during Covid-19 Outbreak in Northern Italy. N Engl J Med. 2020.

2. Reed ME, Huang J, Parikh R, Millman A, Ballard DW, Barr I, et al. Patient-Provider Video Telemedicine Integrated With Clinical Care: Patient Experiences. Ann Intern Med. 2019;171(3):222-4.

3. Timpel P, Oswald S, Schwarz PEH, Harst L. Mapping the Evidence on the Effectiveness of Telemedicine Interventions in Diabetes, Dyslipidemia, and Hypertension: An Umbrella Review of Systematic Reviews and MetaAnalyses. J Med Internet Res. 2020;22(3):e16791.

4. Daniel H, Sulmasy LS, Health and Public Policy Committee of the American College of Physicians. Policy recommendations to guide the use of telemedicine in primary care settings: an American College of Physicians position paper. Ann Intern Med. 2015;163(10):787-9.

5. Wilder-Smith A, Chiew CJ, Lee VJ. Can we contain the COVID-19 outbreak with the same measures as for SARS? Lancet Infect Dis. 2020.

6. Centers for Disease Control and Prevention (CDC): Outpatient and Ambulatory Care Settings: Responding to Community Transmission of COVID-19 in the United States. Retrieved from https://www.cdc.gov/ coronavirus/2019-ncov/hcp/ambulatory-care-settings.html. Accessed June 5, 2020.

7. Centers for Disease Control and Prevention (CDC): Framework for Healthcare Systems Providing Non-COVID-19 Clinical Care During the COVID-19 Pandemic. Retrieved from https://www.cdc.gov/coronavirus/ 2019-ncov/hcp/framework-non-COVID-care.html. Accessed June 5, 2020.

Publisher's Note: Springer Nature remains neutral with regard to jurisdictional claims in published maps and institutional affiliations. 